\title{
Adiponectin, Gut Hormones, and Insulin Resistance
}

\author{
Michael Trakhtenbroit • Heinrich Taegtmeyer
}

Published online: 14 September 2010

(C) Springer Science+Business Media, LLC 2010

We are pleased to see confirmation of our findings on changes in adiponectin and insulin after bariatric surgery in the recent meta-analysis by Butner and colleagues [1]. We would like to emphasize the importance of long-term follow-up that the authors draw upon in their conclusion and its effect on adipokine levels after bariatric surgery. In our study, no significant difference in adiponectin levels between Roux-en-Y gastric bypass and adjustable gastric banding patients was evident until after 9 months [2]. Only at 24 months after surgery did a significant correlation emerge between adiponectin levels and insulin resistance [2]. This correlation, however, existed only in our gastric bypass patients.

We would like to address the authors' statement that our study provided no mention of diabetes in our inclusion or exclusion criteria [1]. Exclusion criteria consisted of age less than 18 years, pregnancy, coronary artery disease, peripheral vascular disease, ischemic cardiomyopathy, or a current history of smoking [2]. Diabetic patients participated in our study.

We applaud the authors for their review of data examining the roles of adipokines and gut hormones in the mechanism of weight loss following bariatric surgery. Research into the role of brain-gut crosstalk and enteric hormone production has become increasingly popular and

M. Trakhtenbroit $\cdot H$. Taegtmeyer $(\triangle)$

Department of Internal Medicine, Division of Cardiology,

University of Texas Medical School at Houston,

6431 Fannin, Room MSB 1.246,

Houston, TX 77030, USA

e-mail: Heinrich.Taegtmeyer@uth.tmc.edu clearly plays a crucial role in weight loss and insulin resistance [3]. With the recent popularity and established efficacy of GLP1 analogues in the treatment of type 2 diabetes, new research is attempting to address changes in incretin levels after bariatric surgery to elucidate whether GLP1 or a yet identified intestinal factor is responsible for a reversal of insulin resistance and weight loss $[4,5]$. We agree with the authors' assertion that further long-term research is needed and add that an investigation into GLP1 and its role as a pharmacologic agent to treat obesity is warranted.

Conflicts of Interest The authors have no conflicts of interest related to this issue.

\section{References}

1. Butner KL, Nickols-Richardson SM, Clark SF, et al. A review of weight loss following Roux-en-Y gastric bypass vs restrictive bariatric surgery: impact on adiponectin and insulin. Obes Surg. 2010;20(5):559-68.

2. Trakhtenbroit MA, Leichman JG, Algahim MF, et al. Body weight, insulin resistance, and serum adipokine levels 2 years after 2 types of bariatric surgery. Am J Med. 2009;122:435-42.

3. Patriti A, Facchiano E, Sanna A, et al. The enteroinsular axis and the recovery from type 2 diabetes after bariatric surgery. Obes Surg. 2004; $14: 840-8$.

4. Salinari S, Bertuzzi A, Asnaghi S, et al. First-phase insulin secretion and differential response to glucose load depending on the route of administration in type 2 diabetics subjects after bariatric surgery. Diabetes Care. 2009;32:375-80.

5. DePaula AL, Macedo AL, Schraibman V, et al. Hormonal evaluation following laparascopic treatment of type 2 diabetes mellitus patients with BMI 20-34. Surg Endosc. 2009;23(8):1724-32. 